\title{
The effects of carbon/phosphorus ratio on polyphosphate- and glycogen-accumulating organisms in aerobic granular sludge
}

\author{
A. Muszyński ${ }^{1}$ A. Miłobędzka ${ }^{1}$
}

Received: 14 October 2014/Revised: 3 February 2015/Accepted: 26 April 2015/Published online: 14 May 2015

(C) Islamic Azad University (IAU) 2015

\begin{abstract}
A laboratory-scale granular sequencing batch reactor, fed with acetate, was operated at two different ratios of chemical oxygen demand to phosphorus-15:1 and 100:1. Smaller aerobic granules, but with better settleability, were obtained at the lower ratio. High ratio of phosphorus release to uptake of dissolved organic carbon $(0.42 \mathrm{~mol} / \mathrm{mol})$ coincided with high percentage of polyphosphate-accumulating organisms (up to $70 \%$ of all bacteria) and implied high metabolic activity of these bacteria. Polyphosphate-accumulating organisms belonged mainly to Accumulibacter and Tetrasphaera (46 and $23 \%$, respectively). Despite significant abundance, Tetrasphaera-related microorganisms were not detected by oligoprobes Actino-221 and Actino658, but by broader oligoprobes Tet2-892 and Tet3-654. Low abundance (1\%) of Halomonas phosphatis indicated a minor role of these bacteria in the laboratory-scale reactor fed with synthetic wastewater. When the ratio of chemical oxygen demand to phosphorus was increased to 100:1, deterioration of settling properties was observed, caused by growth of filamentous organisms from Thiothrix/021N group. The higher ratio favoured Competibacter and was selected against all groups of polyphosphate-accumulating organisms. However, a significant percentage $(10 \%)$ of polyphosphate-accumulating organisms in the granular sludge with concomitant low ratio of phosphorus release to
\end{abstract}

Electronic supplementary material The online version of this article (doi:10.1007/s13762-015-0828-8) contains supplementary material, which is available to authorized users.

A. Muszyński

adam.muszynski@is.pw.edu.pl

1 Faculty of Environmental Engineering, Department of Biology, Warsaw University of Technology, 00-653 Warsaw, Poland the uptake of dissolved organic carbon $(0.01 \mathrm{~mol} / \mathrm{mol})$ suggested shift in the overall population metabolism. Under phosphorus limitation in wastewater, polyphosphate-accumulating organisms no longer synthesized poly-P and behaved as glycogen-accumulating organisms.

Keywords Biological phosphorus removal · Competition · Microbial community · Molecular biology

\section{Introduction}

Phosphorus stimulates biological productivity in water bodies and together with nitrogen, at elevated concentrations, leads to eutrophication. There are two major inputs of phosphorus into surface waters-runoffs from agricultural areas and wastewater discharges. Conventional continuously aerated activated sludge systems, designed principally to eliminate organic matter [expressed as chemical oxygen demand (COD) or biological oxygen demand (BOD)], produce effluents with high residual phosphorus level, which do not meet stringent regulations for wastewater discharge (Jenkins and Wanner 2014).

Wastewater treatment systems have evolved to address efficient elimination of nitrogen and phosphorus. One of the worldwide used strategies to decrease phosphorus concentration in effluent is enhanced biological phosphorus removal (EBPR), and another promising alternative option is microalga wastewater treatment systems. The latter suffers from inconsistent light intensity or wavelength, which is the main obstacle to be overcome for successful upscaling of this process (Yan et al. 2013; Yan and Zheng 2014).

EBPR process is based on circulation of activated sludge through anaerobic and aerobic conditions to enrich polyphosphate-accumulating organisms (PAOs). PAOs take 
up readily degradable organic substrates anaerobically and store them as polyhydroxyalkanoates (PHAs) using energy from the cleavage of intracellularly stored polyphosphate (poly-P). In aerobic conditions, PAOs use the stored PHAs for biomass synthesis and excessive phosphate uptake to replenish the depleted poly-P pool. Phosphorus is eliminated by removal of waste activated sludge with high poly-P content (Seviour and Nielsen 2010).

The candidate PAOs include 'Candidatus Accumulibacter phosphatis' (hereafter called Accumulibacter), Tetrasphaera-related organisms (Actinobacteria) and 'Candidatus Halomonas phosphatis' (hereafter called Halomonas) (Seviour and Nielsen 2010; Nguyen et al. 2011, 2012; Jenkins and Wanner 2014). However, glycogen-accumulating organisms (GAOs) are also able to proliferate in EBPR systems. GAOs take up anaerobically organics, but they use glycogen as source of energy, which results in deterioration of EBPR process. Two main groups of GAOs are alphaproteobacterial Defluviicoccus vanus-related bacteria and gammaproteobacterial 'Candidatus Competibacter phosphatis' (hereafter called Competibacter), both competing with PAO for substrates under anaerobic conditions (Seviour and Nielsen 2010; Jenkins and Wanner 2014).

Aerobic granular sludge (AGS) is an attractive alternative for flocculent activated sludge. This technology provides excellent settleability, high biomass concentration, retention of slow-growing microorganisms and resistance to loading and toxic shocks. Therefore, it can be successfully used not only for nitrogen and phosphorus removal (Wang et al. 2014; Wei et al. 2014), but also for industrial wastewater treatment (Bumbac et al. 2015) and toxic organic compound degradation (Zhu et al. 2014). Community structure in aerobic granules is one of the most interesting topics for researchers (de Kreuk and van Loosdrecht 2004; de Kreuk et al. 2007; Lee et al. 2010; Weissbrodt et al. 2013).

The efficiency of EBPR is strongly dependent on the availability of appropriate carbon sources (Drewnowski and Makinia 2014). COD/P ratio affects the structure of bacterial communities involved in EBPR; however, majority of studies on PAO-GAO selection have been carried out on flocculent activated sludge as reviewed by Oehmen et al. (2007). Very little knowledge is available on the PAO-GAO competition in AGS (de Kreuk and van Loosdrecht 2004; Lemaire et al. 2008; Ahn et al. 2009; Winkler et al. 2011; Weissbrodt et al. 2013). Lots of surveys focused on factors that influence granule formation and stability (Lee et al. 2010).

This research provides important information for understanding the structure of microbial communities in AGS involved in phosphorus removal. Changes in bacterial abundance were monitored using the recently developed oligonucleotide probes for PAOs and GAOs, including those targeting Tetrasphaera-related PAOs (Nguyen et al. 2011), Halomonas (Nguyen et al. 2012) and
Defluviicoccus-related cluster 3 (Nittami et al. 2009). It was proved that formation of strong granules and performance of the granular sludge reactor were strongly affected by $\mathrm{COD} / \mathrm{P}$ ratio. $\mathrm{PAO}$ and GAO selection in AGS was significantly influenced by the availability of phosphorus in wastewater in relation to COD. The obtained results provide useful knowledge of factors that govern PAO-GAO competition in AGS and could be crucial to promote effective phosphorus removal based on granular sludge technology. The research was carried out from October 2012 to July 2013 at Department of Biology, Warsaw University of Technology, Poland.

\section{Materials and methods}

\section{Reactor operation and wastewater}

A laboratory-scale granular sequencing batch reactor (GSBR) with a working volume of 6.91 (Muszyński et al. 2013) was inoculated with activated sludge from a fullscale wastewater treatment plant (WWTP) in Babice (near Warsaw, Poland). The WWTP was configured as anaerobic-anoxic-aerobic (A2O) process with a pre-denitrification step and treated typical municipal wastewater (corresponding to 6000 population equivalent), achieving efficient EBPR. An average influent had COD of $1100 \mathrm{mg}$ $\mathrm{O}_{2} / \mathrm{l}$, a total $\mathrm{P}$ of 23 and $\mathrm{N}$ of $83 \mathrm{mg} / \mathrm{l}$. Average values of COD, total $\mathrm{P}$ and $\mathrm{N}$ for effluent were 34,14 and $0.3 \mathrm{mg} / \mathrm{l}$, respectively (all data provided by the plant operator).

The GSBR was operated for over 8 months. Initially the reactor cycle consisted of an anoxic/anaerobic period of $120 \mathrm{~min}$ (including $10 \mathrm{~min}$ of filling), an aerobic period of $190 \mathrm{~min}$, a settling period of $40 \mathrm{~min}$ and a decantation period (half of the working volume) of $10 \mathrm{~min}$. AGS was cultured by shortening the settling time from 40 to $6 \mathrm{~min}$ (by 2 min per day) and extending the aerobic period to $224 \mathrm{~min}$. This resulted in a 6-h cycle and a hydraulic retention time (HRT) of $12 \mathrm{~h}$. At the end of the aerobic period, the excess sludge was withdrawn once a day as mixed liquor to maintain solids' retention time (SRT) of 8-18 days and mixed liquor suspended solids (MLSS) of 3-4 g/l. The dissolved oxygen concentration was maintained at $2-4 \mathrm{mg} / \mathrm{l}$ during the aerobic period, while during the anoxic/anaerobic phase, the reactor content was mechanically stirred (40 rpm).

The GSBR was operated in two runs with different COD/P ratios in the feed-15:1 (first run) and 100:1 (second run). The feed to the GSBR was similar to the synthetic medium used by Lu et al. (2006). It was prepared daily using stock solutions and contained ( $\mathrm{mg}$ per litre): $770 \mathrm{CH}_{3} \mathrm{COONa}, 1.5$ peptone, 1.5 yeast, $153 \mathrm{NH}_{4} \mathrm{Cl}, 180$ $\mathrm{MgSO}_{4} \cdot 7 \mathrm{H}_{2} \mathrm{O}, 21.5 \mathrm{CaCl}_{2}, 0.9 \mathrm{FeCl}_{3} \cdot 6 \mathrm{H}_{2} \mathrm{O}, 0.09 \mathrm{H}_{3} \mathrm{BO}_{3}$, 
$0.018 \mathrm{CuSO}_{4} \cdot 5 \mathrm{H}_{2} \mathrm{O}, 0.108 \mathrm{KI}, 0.072 \mathrm{MnCl}_{2} \cdot 4 \mathrm{H}_{2} \mathrm{O}, 0.036$ $\mathrm{Na}_{2} \mathrm{MoO}_{4} \cdot 2 \mathrm{H}_{2} \mathrm{O}, 0.072 \mathrm{ZnSO}_{4} \cdot 7 \mathrm{H}_{2} \mathrm{O}, 0.09 \mathrm{CoCl}_{2} \cdot 6 \mathrm{H}_{2} \mathrm{O}$ and 6 EDTA. Concentrations of $\mathrm{K}_{2} \mathrm{HPO}_{4}$ and $\mathrm{KH}_{2} \mathrm{PO}_{4}$ were 112 and $88 \mathrm{mg} / \mathrm{l}$ in the first run and 16.8 and $12.2 \mathrm{mg} /$ 1 in the second run. The GSBR was operated for 171 and 94 days in the first and second runs, respectively, to achieve steady-state conditions without marked changes in effluent parameters within 2 weeks. On the last day of each run (in steady-state conditions), anaerobic phosphorus release $\left(\mathrm{P}_{\text {rel }}\right)$ and dissolved organic carbon uptake (DOC $\left.\mathrm{upt}_{\mathrm{ut}}\right)$ tests were performed. For this purpose, orthophosphate $\left(\mathrm{PO}_{4}-\mathrm{P}\right)$ and DOC concentrations in the GSBR have been measured every $10 \mathrm{~min}$ during the anaerobic phase. FISH analyses and poly-P granule visualizations were performed for seeding sludge and AGS in the GSBR on the last day of each run. Properties of flocs and granules had been examined weekly using light microscopy.

\section{Microscopic analyses}

FISH analyses were carried out to investigate PAO and GAO populations in seeding sludge and AGS in the GSBR. All procedures were performed according to Nielsen et al. (2009). The 6-Fam-labelled general EUBmix probe (equimolar mixture of EUB338, EUB338II and EUB338III) was used to target the entire bacterial community. For PAO detection, the specific probes were applied as follows: PAOmix (PAO462, PAO651 and PAO846) targeting most Accumulibacter, Acc-I-444 (Accumulibacter clades IA and others), Acc-II-444 (Асcumulibacter clades IIA, IIC and IID), Hal180 (Halomonas), Actino-221 (Tetrasphaera Type 1), Actino-658 (Tetrasphaera type 2) and Tet1-266, Tet2-892, Tet2-174, Tet3654 targeting Tetrasphaera clades 1, 2A, 2B and 3, respectively. The more broadly specific Pse136 probe was applied in a hierarchal approach to decrease the possibility of false positives for probe Hal180. GAOs were identified with specific probes: GB targeting most Competibacter, DF1mix (TFO_DF218 and TFO_DF618), DF2mix (DF988 and DF1020) and DF198 targeting Defluviicoccus vanusrelated TFO clusters 1, 2 and 3, respectively. G123T probe was used for detection of Thiothrix/021N group. All specific probes were labelled with $\mathrm{Cy} 3$. Detailed information about the probes used is given in probeBase (Loy et al. 2003). Twenty separate images for each probe used were captured with a Nikon Eclipse 50i microscope, and percentage abundance of specific bacteria was then calculated as described by Muszyński et al. (2013). Presence of poly-P granules in bacterial cells was tested by DAPI staining ( $1 \mu \mathrm{g} / \mathrm{ml}, 1 \mathrm{~h})$ as described by Zilles et al. (2002).

Visual characterization of sludge samples was performed using Nikon Eclipse 80i microscope. Size of sludge flocs and granules was measured using NIS-Elements AR
(Nikon) software and was expressed as means of 100 separate measurements for length and width.

\section{Chemical analyses}

DOC was measured by a TOC analyzer TOC-5000 (Shimadzu, Japan). Soluble orthophosphate $\left(\mathrm{PO}_{4}-\mathrm{P}\right)$ and COD were determined spectrophotometrically by the use of standard LCK vial test kits (HACH-Lange). Sludge volume index (SVI), mixed liquor suspended solid (MLSS) and mixed liquor volatile suspended solid (MLVSS) weight were determined at the end of the aerobic periods in accordance with Standard Methods (1999).

\section{Results and discussion}

\section{Formation and characteristics of granules in response to different $\mathrm{COD} / \mathrm{P}$ ratios}

The GSBR was seeded with flocculent activated sludge. First, irregular granules (138 \pm 6 and $49 \pm 8 \mu \mathrm{m}$ of length and width, respectively) were observed after 2 weeks of operation in the first run. No single mechanism is responsible for formation of aerobic granules - this process is strongly influenced by different parameters and factors, like substrate composition, organic loading rate, feeding strategy (feast and famine periods), reactor design, hydrodynamics, settling time, exchange ratio and aeration intensity (Lee et al. 2010). However, a critical parameter seems to be short settling time required to retain particles with good settleability. Lemaire et al. (2008) operated a laboratory-scale GSBR for 870 days with no granule formation before shortening the settling time from 40 to $5 \mathrm{~min}$. In research presented in this article, first granules appeared after decreasing the settling time to $12 \mathrm{~min}$. Their size successively increased to $296 \pm 13 \mu \mathrm{m}$ of length and $244 \pm 14 \mu \mathrm{m}$ of width after 2 months when the settling time was gradually decreased to $6 \mathrm{~min}$. The length did not change significantly till the end of the first run, but the granules became more dense and regular-the aspect ratio changed from 3 to about 1.2. The settling properties improved, and SVI decreased from $140 \mathrm{ml} / \mathrm{g}$ (seeding sludge) to below $30 \mathrm{ml} / \mathrm{g}$ on day 171. Ahn et al. (2009) showed that dimensions and structure of aerobic granules in a laboratory-scale anaerobic-aerobic GSBR strictly depended on $\mathrm{pH}$ value. When $\mathrm{pH}$ fell from 7.5 to 6.5 , the granules increased in size from 200 to $2000-3000 \mu \mathrm{m}$ and were regularly spherical with smooth outer surfaces.

When the COD/P ratio was increased from 15:1 to $100: 1$ in the second run, the granules got bigger $(420 \pm 22 \mu \mathrm{m}$ in width and $544 \pm 19 \mu \mathrm{m}$ in length), but deterioration of the settling properties was observed (SVI increased to $95 \mathrm{ml} / \mathrm{g}$ 
on day 94 of the second run). A similar effect was observed by Lin et al. (2003) - the size of granules exhibited a decreasing trend with the decrease in substrate COD/P ratio, while the structure of granules became more compact and denser resulting in lower SVI of microbial granules. The results of the present research confirmed that more compact aggregates are obtained at lower COD/P ratios. It should be mentioned that SVI of the granules in the second run of this study was in the range $50-100 \mathrm{ml} / \mathrm{g}$, reported for non-Paccumulating aerobic granules (Lin et al. 2003). Sedimentation of biomass took place after the aeration period; therefore, granules dominated by PAOs contained high amounts of poly-P, which improved settling properties in comparison with GAO-dominated granules. PAOs are prevalent at the bottom of reactors, whereas GAOs dominate at the top. Based on this observation, Winkler et al. (2011) proposed selective sludge removal from GSBR as a strategy to control PAO-GAO competition. In the present study, excess sludge was withdrawn at the end of the aerobic period from the middle part of the GSBR, to avoid applying another parameter (apart from COD/P ratio in the influent) influencing PAO and GAO communities.

The deterioration of settling properties, observed in the second run, was mainly due to growth of filamentous organisms. The granules exhibited characteristic filamentous outer structure (rosettes) despite maintaining the same operational parameters except for lower $\mathrm{P}$ concentration in the influent. Filamentous bacteria belonged to Thiothrix/ $021 \mathrm{~N}$ and accounted for $7 \pm 3 \%$ of all bacteria identified by probe EUBmix on the last day of the second run, whereas they were not detected in the first run neither in the seeding sludge. Thiothrix were dominating filamentous bacteria in GSBR treating brewery wastewater; the granules were extremely big and fluffy, and they did not settle properly and caused bulking problems in the reactor (Weber et al. 2007). However, Li et al. (2014) found Thiothrix as dominant filamentous bacteria in aerobic granules without negative impact on settling velocity. Several other studies also reported deterioration of settling properties of AGS, associated with overgrowth of filamentous bacteria (Liu and Liu 2006; Weissbrodt et al. 2012).

\section{Changes in PAO-GAO population: first run (COD/ $P=15: 1)$}

The fraction of PAOs in the seeding sludge accounted for $37 \%$ of all bacteria, and it was comprised mainly of Tetrasphaera PAO (33\%), whereas abundance of Accumulibacter was much lower (4\%)-Fig. 1. Neither Halomonas nor clade II of Accumulibacter were detected in the seed, but they were present in GSBR granules later in the first run. On the other hand, clade 1 of
Tetrasphaera accounted for $5 \%$ of bacterial biovolume in the seeding sludge, but it was not detected in the first run nor the second run of the GSBR. The GSBR was fed with acetate as the single carbon source, whereas clade 1 of Tetrasphaera was not shown to take up this substrate (Nguyen et al. 2011). In full-scale systems, Tetrasphaerarelated PAOs are usually more abundant than Accumulibacter, but in laboratory-scale reactors, those bacteria were overlooked (Nguyen et al. 2011). However, two actinobacterial probes Actino-221 and Actino-658, which had been widely used (Kong et al. 2005), cover only a part of the three Tetrasphaera clades (Nguyen et al. 2011). GAOs in the seeding sludge did not exceed $2 \%$ of EUBmix, and they were represented only by Competibacter.

Continuous operation of the GSBR in the first run resulted in good, stable COD and phosphorus removal (85-96\% and 98-99\%, respectively) and low concentrations in the effluent (29-75 $\mathrm{mg} \mathrm{COD} / \mathrm{l}$ and $0.2-0.8 \mathrm{mg} \mathrm{P} / \mathrm{l})$. Chemical profiles of extracellular $\mathrm{P}^{-} \mathrm{PO}_{4}{ }^{3-}$ and DOC concentrations on day 171 were consistent with the PAO phenotype (Fig. 2). The $\mathrm{P}_{\text {rel }} /$ $\mathrm{DOC}_{\mathrm{upt}}$ ratio $(0.42 \mathrm{~mol} / \mathrm{mol})$ fell within the range $(0.30-0.75 \mathrm{~mol} / \mathrm{mol})$ reported in the literature ( $\mathrm{Lu}$ et al. 2006; Oehmen et al. 2007) for Accumulibacter and acetate. It implies high activity of PAOs at the end of the first run. The accumulation of poly-P was confirmed by DAPI staining of biomass sampled at the end of the aerobic period as shown in Online Resource 1 (micrographs A and B). The FISH analyses revealed that nearly $70 \%$ of all bacteria detected in granules were putative PAOs (Fig. 1). Halomonas PAOs played a minor role in AGS in this survey, as detected in low abundance $(1 \%$ of EUBmix) and only in the first run. Accumulibacter constituted $46 \%$ of all bacteria, whereas Tetrasphaera clades (mainly 3 and 2A) were half as abundant as Accumulibacter was, as presented in Online Resource 2 (micrographs A and C). It may be concluded that low COD/P ratio promotes growth of PAOs, which belong mainly to Accumulibacter and, to less extent, to Tetrasphaera.

Weissbrodt et al. (2013) proved that Accumulibacter selection in AGS can be favoured in the presence of propionate when compared to acetate only (25-40\% and $6 \%$ of EUBmix, respectively). A significant percentage (up to $16 \%)$ of Tetrasphaera was also detected in a laboratoryscale GSBR fed with acetate, but abundance of this putative PAOs progressively decreased to less than $5 \%$ when propionate was used alone or in mixture with acetate (Weissbrodt et al. 2013). Ahn et al. (2009) detected Tetrasphaera-related PAOs with Actino-658 probe after starting GSBR only, which was inoculated with activated sludge from full-scale WWTP. Those PAOs were rarely 
Fig. 1 Abundance of putative PAOs and GAOs (mean values and standard errors) determined by qFISH in seed and sludge on days $171(\mathrm{COD} / \mathrm{P}=15: 1)$ and $94(\mathrm{COD} / \mathrm{P}=100: 1)$ of the first and second runs, respectively. Tetrasphaera clade 2B accounted for a minor fraction $(<0.1 \%)$; Defluviicoccus vanus-related TFO clusters 2 and 3 were not detected and are not shown
Fig. 2 Profiles of extracellular $\mathrm{P}_{-} \mathrm{PO}_{4}{ }^{3-}$ and DOC during the anaerobic period of the GSBR on days $171(\mathrm{COD} / \mathrm{P}=15: 1)$ and $94(\mathrm{COD} / \mathrm{P}=100: 1)$ of the first and second runs. Means of three replicates are presented, and error bars illustrate standard errors. Error bars that are smaller than the marker symbols are not shown
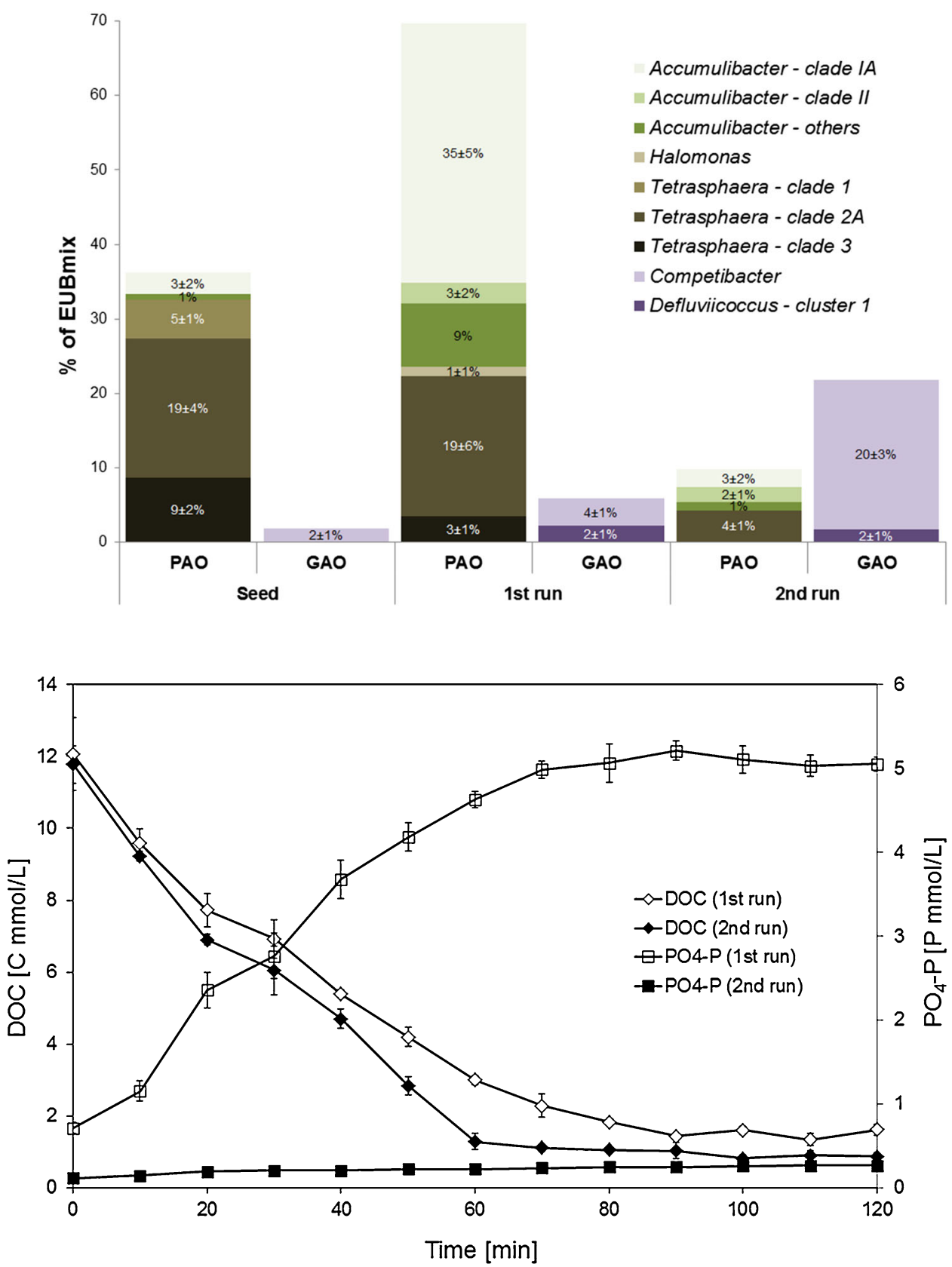

seen in subsequent samples, probably due to inability of Actino-658-targeted Tetrasphaera to utilize acetate. In this research, Tetrasphaera-related PAOs were not detected in the GSBR with the probes Actino-658 and Actino-221 either, although those putative PAOs were present in the seed in abundance of 0.1 and $9 \%$, respectively (data not shown). However, the presence of Tetrasphaera in GSBR was confirmed with the broader probes designed by Nguyen et al. 2011. The controversy regarding the ability of Tetrasphaera PAOs to utilize acetate (Kong et al. 2005; Nguyen et al. 2011) may be explained by the broad coverage of the latter probes (Nguyen et al. 2011).
The abundance of Competibacter GAOs in the first run did not change significantly in comparison with the seeding sludge and did not exceed $4 \%$ of the biovolume, as shown in Online Resource 2 (micrograph E). It can be concluded that low COD/P ratio did not affect the growth of Competibacter GAOs. However, despite the absence of Defluviicoccus vanus-related TFOs from cluster 1 in the seed, these microorganisms appeared in granules $(2 \%$ of EUBmix). This group of GAOs is rarely detected in fullscale WWTPs; however, it is more frequently observed in laboratory-scale reactors. Significant abundance (2-6\%) of Defluviicoccus cluster 2 was found in GSBR by Ahn 
et al. (2009), but these GAOs were not detected in AGS by Lemaire et al. (2008) nor in this study.

\section{Changes in PAO-GAO population: second run $(\mathrm{COD} / \mathrm{P}=100: 1)$}

Chemical profiles of extracellular $\mathrm{P}_{-} \mathrm{PO}_{4}{ }^{3-}$ and DOC concentrations on day 94 of the second run showed a distinct shift from PAO to GAO phenotype (Fig. 2). A higher $\mathrm{COD} / \mathrm{P}$ ratio resulted in a significantly lower $\mathrm{P}-\mathrm{PO}_{4}{ }^{3-}$ release. However, the organic carbon uptake rates in the second and first runs were similar (20 and $23 \mathrm{mg}$ DOC/g $\mathrm{MLSS} / \mathrm{h}$, respectively), which resulted in distinctly lower $\mathrm{P}_{\text {rel }} / \mathrm{DOC}_{\mathrm{upt}}$ ratio in the second run $(0.01 \mathrm{~mol} / \mathrm{mol})$. Deficiencies in poly-P accumulation were visualized by DAPI staining-hardly any polyphosphate-positive cells were present in biomass sampled at the end of the aerobic period (Online Resource 1, micrographs C and D). It coincided with marked increase in MLVSS/MLSS ratio from $51 \%$ (first run) to $88 \%$ (second run). A sharp decline in abundance of all PAO groups was observed (Online Resource 2, micrographs B and D), while the content of Competibacter increased from 4 to $20 \%$ (Online Resource 2, micrograph F). A percentage of overall PAO community in the whole PAO and GAO microbiomes, expressed as PAOs/ (PAOs+GAOs), dropped from 92 to $31 \%$ (first and second runs, respectively)-Fig. 1. De Kreuk and van Loosdrecht (2004) showed by semi-quantitative FISH analysis (subjective approximation) that PAO community in GSBR can be almost completely replaced by GAOs when dosage of phosphate is stopped. Similar changes in abundance of Rhodocyclus-related PAOs, but in flocculent sludge, were observed by Kong et al. (2002) in a laboratory-scale reactor fed with acetate as a sole carbon source.

The results of the present study, concerning the higher $\mathrm{PAO} / \mathrm{GAO}$ level in GSBR fed with lower COD/P ratios, are inconsistent with the findings of Weissbrodt et al. (2013). They concluded that higher ratios of Accumulibacter to Competibacter can be obtained with higher $\mathrm{COD} / \mathrm{P}$, whereas lower COD concentrations favour mainly Competibacter and Tetrasphaera. However, COD/P ratio was not highlighted by ANOVA as a significant factor of the PAO/GAO level response-mainly temperature, $\mathrm{pH}$ and full aerobic conditions during starvation impacted the Accumulibacter/Competibacter competition (Weissbrodt et al. 2013). Lemaire et al. (2008) showed that Accumulibacter-Competibacter stratification in aerobic granules is strongly correlated with the dissolved oxygen concentration gradient. Accumulibacter are abundant in the outermost region of the granule, while Competibacter dominate the granule central zone.

The complete domination of AGS by GAO community in this study was ruled out by FISH analyses; apart from GAOs (22\% of EUBmix), also PAOs were present in significant abundance (up to $10 \%$ ) in granules. However, the $\mathrm{P}_{\text {rel }} / \mathrm{DOC}_{\text {upt }}$ ratio $(0.01 \mathrm{~mol} / \mathrm{mol})$, determined on day 94 of the second run, was close to zero which is typical for highly enriched GAO cultures (>90\%) (Lu et al. 2006; Oehmen et al. 2007). This research proved that under extended phosphorus limitation in wastewater, PAOs present in AGS no longer synthesize poly-P, as it was shown by DAPI staining, and behave as GAOs, which was distinctly presented with chemical profiles. Therefore, decreases in EBPR capacity may not necessarily reflect shifts in community composition, but in the existing population metabolism to GAO phenotype. Similarly, Barat et al. (2008) and Zhou et al. (2008) observed that under poly-P-limited conditions, the highly enriched PAO culture is able to take up acetate using glycogen as the primary energy source.

\section{Conclusion}

Aerobic granules were formed at different $\mathrm{COD} / \mathrm{P}$ ratios in a laboratory-scale GSBR fed with acetate as a sole carbon source. Characteristics of granules and changes in structure of PAO and GAO communities were investigated. Smaller aggregates, but with better settleability, were obtained at lower COD/P ratio (15:1). Higher COD/P ratio (100:1) induced bigger size of granules, but also deteriorated settling properties, mainly due to growth of filaments from Thiothrix/021N group. Lower COD/P ratio in the feed was selected for PAOs, which belonged mainly to Accumulibacter and Tetrasphaera clades (46 and $23 \%$ of all bacteria, respectively); the role of Halomonas in EBPR was minor (1\%). Despite significant abundance, Tetrasphaerarelated PAOs were not detected with probes Actino-221 and Actino-658, but with the broader probes Tet2-892 and Tet3-654. Therefore, the broader oligoprobes should be applied to detect Tetrasphaera-related PAOs instead of actinobacterial probes. Marked $\mathrm{P}_{\text {rel }} / \mathrm{DOC}_{\mathrm{upt}}$ ratio $(0.42 \mathrm{~mol} / \mathrm{mol})$ at lower COD/P in the influent coincided with the high percentage of PAOs (up to $70 \%$ of EUBmix probe), whereas higher $\mathrm{COD} / \mathrm{P}$ ratio favoured 
Competibacter GAOs and was selected against all groups of PAOs (abundance 22 and $10 \%$, respectively). However, significant percentage of PAOs in GSBR with concomitant low $\mathrm{P}_{\text {rel }} / \mathrm{DOC}_{\text {upt }}$ ratio $(0.01 \mathrm{~mol} / \mathrm{mol})$ suggested shift in the overall population metabolism. Under extended phosphorus limitation in wastewater, PAOs no longer synthesized poly-P and behaved as GAOs. It implies that PAOs are highly adaptable and may cope with different WWTP conditions.

Acknowledgments The authors recognize the excellent technical assistance of E. Łukomska, D. Rosińska, J. Klimkiewicz and Ł. Juraszek.

\section{References}

Ahn J, McIlroy S, Schroeder S, Seviour R (2009) Biomass granulation in an aerobic:anaerobic-enhanced biological phosphorus removal process in a sequencing batch reactor with varying $\mathrm{pH}$. $\mathrm{J}$ Ind Microbiol Biotechnol 36:885-893

Barat R, Montoya T, Borrás L, Ferrer J, Seco A (2008) Interactions between calcium precipitation and the polyphosphate accumulating bacteria metabolism. Water Res 42:3415-3424

Bumbac C, Ionescu IA, Tiron O, Badescu VR (2015) Continuous flow aerobic granular sludge reactor for dairy wastewater treatment. Water Sci Technol (in press). doi:10.2166/wst.2015.007

Clescerl LS, Greenberg AE, Eaton AD (eds) (1999) Standard Methods for the Examination of Water and Wastewater, 20th edn. American Public Health Association/American Water Works Association/Water Environment Federation, Washington, DC

de Kreuk MK, van Loosdrecht MCM (2004) Selection of slow growing organisms as a means for improving aerobic granular sludge stability. Water Sci Technol 49(11-12):9-17

de Kreuk MK, Kishida N, van Loosdrecht MC (2007) Aerobic granular sludge-state of the art. Water Sci Technol 55(8-9):75-81

Drewnowski J, Makinia J (2014) The role of biodegradable particulate and colloidal organic compounds in biological nutrient removal activated sludge systems. Int J Environ Sci Technol 11:1973-1988

Jenkins D, Wanner J (2014) Activated sludge-100 years and counting. IWA Publishing, London

Kong YH, Beer M, Rees GN, Seviour R (2002) Functional analysis of microbial communities in aerobic-anaerobic sequencing batch reactors fed with different phosphorus/carbon (P/C) ratios. Microbiology 148:2299-2307

Kong YH, Nielsen JL, Nielsen PH (2005) Identity and ecophysiology of uncultured actinobacterial polyphosphate-accumulating organisms in full-scale enhanced biological phosphorus removal plants. Appl Environ Microbiol 71:4076-4085

Lee DJ, Chen YY, Show KY, Whiteley CG, Tay JH (2010) Advances in aerobic granule formation and granule stability in the course of storage and reactor operation. Biotechnol Adv 28:919-934

Lemaire R, Yuan Z, Blackall LL, Crocetti GR (2008) Microbial distribution of Accumulibacter spp and Competibacter spp in aerobic granules from a lab-scale biological nutrient removal system. Environ Microbiol 10:354-363

Li Y, Zou J, Zhang L, Sun J (2014) Aerobic granular sludge for simultaneous accumulation of mineral phosphorus and removal of nitrogen via nitrite in wastewater. Bioresour Technol 154:178-184

Lin YM, Liu Y, Tay JH (2003) Development and characteristics of phosphorus-accumulating microbial granules in sequencing batch reactors. Appl Microbiol Biotechnol 62:430-435

Liu Y, Liu Q-S (2006) Causes and control of filamentous growth in aerobic granular sludge sequencing batch reactors. Biotechnol Adv 24:115-127

Loy A, Horn M, Wagner M (2003) ProbeBase: an online resource for rRNA-targeted oligonucleotide probes. Nucleic Acids Res 31:514-516

Lu H, Oehmen A, Virdis B, Keller J, Yuan ZG (2006) Obtaining highly enriched cultures of Candidatus Accumulibacter phosphatis through alternating carbon sources. Water Res 40:3838-3848

Muszyński A, Łebkowska M, Tabernacka A, Miłobędzka A (2013) From macro to lab-scale: changes in bacterial community led to deterioration of EBPR in lab reactor. Cent Eur $\mathrm{J}$ Biol $8(2): 130-142$

Nguyen HT, Le VQ, Hansen AA, Nielsen JL, Nielsen PH (2011) High diversity and abundance of putative polyphosphate-accumulating Tetrasphaera-related bacteria in activated sludge systems. FEMS Microbiol Ecol 76:256-267

Nguyen HT, Nielsen JL, Nielsen PH (2012) 'Candidatus Halomonas phosphatis', a novel polyphosphate-accumulating organism in full-scale enhanced biological phosphorus removal plants. Environ Microbiol 14:2826-2837

Nielsen PH, Daims H, Lemmer H (2009) Fish handbook for biological wastewater treatment. IWA Publishing, London

Nittami T, Mcllroy S, Seviour EM, Schroeder S, Seviour RJ (2009) Candidatus Monilibacter spp, common bulking filaments in activated sludge, are members of Cluster III Defluviicoccus. Syst Appl Microbiol 32:480-489

Oehmen A, Lemos PC, Carvalho G, Yuan Z, Keller J, Blackall LL, Reis MAM (2007) Advances in enhanced biological phosphorus removal: from micro to macro scale. Water Res 41:2271-2300

Seviour RJ, Nielsen PH (2010) Microbial ecology of activated sludge. IWA Publishing, London

Wang R, Peng Y, Cheng Z, Ren N (2014) Understanding the role of extracellular polymeric substances in an enhanced biological phosphorus removal granular sludge system. Bioresour Technol 169:307-312

Weber S, Ludwig W, Schleifer KH, Fried J (2007) Microbial composition and structure of aerobic granular sewage biofilms. Appl Environ Microbiol 73:6233-6240

Wei D, Shi L, Yan T, Zhang G, Wang Y, Du B (2014) Aerobic granules formation and simultaneous nitrogen and phosphorus removal treating high strength ammonia wastewater in sequencing batch reactor. Bioresour Technol 171:211-216

Weissbrodt DG, Lochmatter S, Ebrahimi S, Rossi P, Maillard J, Holliger C (2012) Bacterial selection during the formation of early-stage aerobic granules in wastewater treatment systems operated under wash-out dynamics. Front Microbiol 3:332

Weissbrodt DG, Schneiter GS, Fürbringer JM, Holliger C (2013) Identification of trigger factors selecting for polyphosphate- and glycogen-accumulating organisms in aerobic granular sludge sequencing batch reactors. Water Res 47:7006-7018 
Winkler MK, Bassin JP, Kleerebezem R, de Bruin LM, van den Brand TP, van Loosdrecht MC (2011) Selective sludge removal in a segregated aerobic granular biomass system as a strategy to control PAO-GAO competition at high temperatures. Water Res 45:3291-3299

Yan C, Zheng Z (2014) Performance of mixed LED light wavelengths on biogas upgrade and biogas fluid removal by microalga Chlorella sp. Appl Energy 113:1008-1014

Yan C, Zhang L, Luo X, Zheng Z (2013) Effects of various LED light wavelengths and intensities on the performance of purifying synthetic domestic sewage by microalgae at different influent $\mathrm{C} / \mathrm{N}$ ratios. Ecol Eng 51:24-32
Zhou Y, Pijuan M, Zeng RJ, Lu H, Yuan Z (2008) Could polyphosphate-accumulating organisms (PAOs) be glycogenaccumulating organisms (GAOs)? Water Res 42:2361-2368

Zhu L, Dai X, Xu X, Lv M, Cao D (2014) Microbial community analysis for aerobic granular sludge reactor treating high-level 4-chloroaniline wastewater. Int $\mathbf{J}$ Environ Sci Technol 11:1845-1854

Zilles JL, Hung CH, Noguera DR (2002) Presence of Rhodocyclus in a full-scale wastewater treatment plant and their participation in enhanced biological phosphorus removal. Water Sci Technol 46(1-2):123-128 